\title{
Type-1 diabetes revisited and lessons learned
}

\author{
Authors: Andrew Brooks, ${ }^{1}$ Jimmy Chong, ${ }^{1}$ David Schpira, ${ }^{1}$ Eleri Williams, ${ }^{1}$ Heather Barbour, ${ }^{1}$ Radhi Krishnan $^{2}$ and \\ Alasdair Warwick ${ }^{2}$
}

\section{Aims}

To re-evaluate current concepts of the natural history of type 1 diabetes and its complications.

\section{Methods}

The carefully assembled clinical records, and accompanying laboratory data of a cohort of white Caucasian patients with type 1 diabetes (T1DM) collected over 25 years have been subject to retrospective analyses. Data were gathered from patients at routine 4- to 12-monthly clinic attendances, entered into an electronic database, and analysed numerically and statistically.

\section{Results}

The number, sex ratio, mean and ranges of age at diagnosis (years) for the calendar decades a) 1961-70; b) 1971-80; c) 1981-90; d) 1991-2000; and e) 2000-10 were respectively: a) 66, $0.74,18.5$ and $0.7-53.5$; b) $114,1.19,18.2$ and $1.3-52.5$; c) 156 , $1.00,23.6$ and $0.2-62.1$; d) $164,0.93,26.9$ and $0.17-73.1$; and e) $194,1.55,21.7$ and $1.3-68.3$.

The numbers and percentages (\%) of patients with a firstdegree relative with diabetes in these decades were respectively: a) $7 / 66,10.6 \%$; b) $9 / 118,7.6 \%$; c) $27 / 158,17.1 \%$; d) $21 / 164$, $12.8 \%$; e) $24 / 194,12.4 \%$.

The percentage (\%) prevalence of comorbid hypothyroidism in males and females of duration i) $0-9.9$; ii) 10-19.9; iii) 20 29.9 ; iv) $30-39.9$; v) $40-49.9$ years were: i) 2.5 and 12.8 ; ii) 9.4 and 15.6 ; iii) 4.4 and 21.8 ; iv) 17.7 and 39.2 ; v) 15.0 and $31.4 \%$. When thyroid function was normal, mean serum TSH levels (miU/L) were 1.94-2.29 and 1.76-2.44 over these 50 years.

Using the same divisions of duration, the mean \pm SD systolic $\mathrm{BP}$ and diastolic BP $(\mathrm{mmHg})$ in untreated males and females were: i) $119 \pm 15,71 \pm 10$ and $116 \pm 14,70 \pm 10$; ii) $122 \pm 13,72 \pm 8$ and $122 \pm 16,74 \pm 8$; iii) $137 \pm 19,76 \pm 13$ and $124 \pm 15,73 \pm 9$; iv) $132 \pm 13$, $76 \pm 11$ and $127 \pm 13,72 \pm 10$; v) $134 \pm 10,74 \pm 6$ and $133 \pm 9,75 \pm 5$.

The $\%$ males and females offered antihypertensive treatment were: i) 7,3 ; ii) 22,8 ; iii) 29,23 ; iv) 30,6 ; v) 35,55 .

Authors: ${ }^{1}$ Hampshire Hospitals NHS Foundation Trust, Winchester SO22 5DG, UK; ${ }^{2}$ Diabetic Eye Screening Service, University of Southampton Hospitals, UK
The $\%$ prevalence of any diabetic retinopathy (R1), proliferative retinopathy (R3) and maculopathy (M1) for durations i) $0-9.9$; ii) $10-19.9$; iii) $20-29.9$; iv) $\geq 30$ years were: i) $39,0,<1$; ii) $68,4,15$; iii) $74,8,17$; iv) $73,16,11 \%$. R3 relates significantly to duration (OR $1.08 /$ year, $\mathrm{p}=0.001$ ) but not patient age (OR 1.01/year, $\mathrm{p}=0.78$ ).

\section{Conclusions}

The age at diagnosis of T1DM has increased, and one in six new-onset patients are over 40 years old. One in eight patients have a first-degree relative with diabetes, which may indicate a subtype. Prevalence of comorbid hypothyroidism increases with age. BP rises with duration of T1DM, with those over 20 years most at risk. The natural history of proliferative retinopathy (R1) and maculopathy (M1) differ in T1DM, with $\mathrm{R} 3$ related to duration of diabetes rather than age of the patient at screening. 